HT2007-32734

\title{
DIAMETER AND LENGTH EFFECT ON DIFFUSIVE-BALLISTIC PHONON TRANSPORT IN A CARBON NANOTUBE
}

\author{
Junichiro Shiomi \\ Department of Mechanical Engineering \\ The University of Tokyo \\ 7-3-1 Hongo, Bunkyo-ku, \\ Tokyo, 113-8656, Japan
}

\author{
Shigeo Maruyama \\ Department of Mechanical Engineering \\ The University of Tokyo \\ 7-3-1 Hongo, Bunkyo-ku, \\ Tokyo, 113-8656, Japan
}

\begin{abstract}
We report a non-equilibrium molecular dynamics (MD) study on heat conduction of finite-length single-walled carbon nanotubes (SWNTs). The length and diameter dependences of the thermal conductivity are quantified for a range of nanotubelengths up to a micrometer at room temperature using two different temperature control techniques. A thorough investigation was carried out on the influence of intrinsic thermal boundary resistance between the temperaturecontrolled layers and the rest of the SWNT. The trend of length effect indicates a gradual transition from nearly pure ballistic phonon transport to diffusive-ballistic phonon transport. The nearly pure ballistic phonon transport was also confirmed by the minor diameter-dependence of thermal conductivity for short SWNTs. For longer SWNTs with stronger diffusive effect, the thermal conductivity is larger for SWNTs with smaller diameters.
\end{abstract}

\section{INTRODUCTION}

The ever-expanding expectations for single-walled carbon nanotubes (SWNTs) include applications for various electrical and thermal devices due to their unique electrical and thermal properties [1]. SWNTs are expected to possess high thermal conductivity due to their strong carbon bonds and the quasione-dimensional structure [2]. On considering the actual applications, one of the essential tasks is to characterize the thermal properties not only for thermal devices but also for electrical devices since they determine the affordable amount of electrical current through the system.

With advances in SWNT synthesis and MEMS techniques, thermal conductivity (or thermal conductance) measurements of individual SWNTs have been recently reported [3, 4]. Measurements were made for 2.76- $\mu \mathrm{m}$-long SWNT suspended across a gap between two thermal reservoirs and thermal conductance of $4 \mathrm{nW} / \mathrm{K}$ was obtained at room temperature [3]. Later, thermal conductivity of a $2.6-\mu \mathrm{m}-$ long individual suspended SWNT with diameter of about $1.7 \mathrm{~nm}$ was extracted from I-V electrical characteristics to be about $3500 \mathrm{~W} / \mathrm{m} \mathrm{K}$ at room temperature. The value is similar to those of individual multi-walled carbon nanotubes $[5,6]$ and about an order of magnitude larger than that of bulk carbon nanotubes in forms of mats and bundles [7]. Experiments with temperature variation suggest that the thermal conductivity (or conductance) increases with temperature in temperature range between $110 \mathrm{~K}$ and $300 \mathrm{~K} \mathrm{[3]}$ and it decreases in temperature range between $300 \mathrm{~K}$ and $800 \mathrm{~K}$ [4]. These observations suggest a critical temperature $(\sim 300 \mathrm{~K})$ above which Umklapp scattering becomes important.

The thermal property measurements of SWNTs mentioned above are extremely challenging as there are potential uncertainties residing in the technicality for instance related to the contact resistances between thermal reservoirs and an SWNT. Therefore, the demands for reliable theories and numerical simulations are greater than ever for validations of the experimental results and for investigation of detail heatconduction characteristics that are not accessible in experiments. One of such heat conduction characteristics with practical importance is the size dependence of thermal conductivity. In general, the size-dependence of the thermal conductivity appears when the system characteristic length is smaller or comparable to the phonon mean free path [8]. For SWNTs, due to the expected long phonon mean free path, the regime of the length effect stretches beyond the realistic length in many applications. The length effect has been demonstrated using MD simulations $[9,10]$ and the power-law divergence was discussed with analogy to the convectional one- 
dimensional models [11]. More recently, the length effect was investigated up to fully diffusive phonon transport regime using a kinetic approach [12], where the divergence was shown to disappear with presence of the second order (or higher) 3phonon scattering processes. The issue of the transition from the pure ballistic to diffusive-ballistic phonon transport was discussed by Wang and Wang [13] by modeling the energy transmission based on the ratio of the overall average phonon mean free path to $L$.

In this study, we aim to investigate phonon transport in SWNTs for a range of SWNT lengths by performing nonequilibrium MD simulations at room temperature. MD simulations are capable of handling phonon transport for all the phonon branches, unlike the kinetic approach with relaxation approximations [12]. As shown later, this aspect is important for SWNTs with significant ballistic phonon transport, especially at room temperature where a wide range of phonon branches is populated.

\section{NOMENCLATURE}

$A=$ nanotube cross-sectional area

$A_{c-c}=$ interatomic distance

$a=$ unit cell thickness

$B^{*}=$ many body term

$E_{b}=$ binding energy

$E=$ phonon energy spectrum

$K=$ thermal conductance

$k=$ wave number

$L=$ nanotube length

$L_{c}=$ thermostat length

$N=$ number of unit cells

$n=$ number of atoms per unit cell

$q=$ heat flux

$r_{i j}=$ distance between atom $i$ and $j$

$V_{A}=$ attractive term

$V_{R}=$ repulsive term

\section{Greek Symbols}

$\alpha=$ cylindrical coordinates

$\Delta T=$ temperature drop

$\lambda=$ thermal conductivity

$\omega=$ frequency

$\tau=$ relaxation time

\section{Subscripts}

$\mathrm{P}=$ phatom thermostat

$\mathrm{NH}=$ Nose-Hoover thermostat

\section{CLASSICAL MOLECULAR DYNAMICS}

\section{The Molecular Dynamics Potential Function}

In current MD simulations, the carbon-carbon interactions were modeled using Brenner potential [14] in a simplified form [15] where the total potential energy of the system is expressed as,
$E_{b}=\sum_{i} \sum_{j(i<j)}\left[V_{R}\left(r_{i j}\right)-B_{i j}^{*} V_{A}\left(r_{i j}\right)\right]$

Here, $V_{R}(r)$ and $V_{A}(r)$ are repulsive and attractive force terms, which take a Morse type form with a certain cut-off function. $B_{i j}^{*}$ represents the effect of the bonding order parameters. As for the potential parameters, we employ the set that was shown to reproduce the force constant better (Table 2 in [14]). The velocity Verlet method was adopted to integrate the equation of motion with the time step of $0.5 \mathrm{fs}$.

The application of classical approach is encouraged by the expected dominant contribution on the heat conduction from phonons compared with that from electrons [16]. While the electric thermal conductance for semi-conducting SWNTs is expected to be negligible, contribution of electric thermal conductance in metallic SWNTs has been calculated to be minor $(\sim 10 \%)$ at room temperature [17].

\section{Dispersion Relations}

On simulating ballistic phonon transport using MD simulations, linear transport properties need to be reproduced with sufficient accuracy. This needs to be satisfied for phonons with a wide range of frequencies as their ballistic transport becomes important for the short SWNTs at room temperature. Linear phonon transport properties can be visualized by the dispersion relations, which can be computed from MD simulations by taking the two-dimensional Fourier spectra of the time history of the one-dimensional velocity field along an SWNT. Here, the spectra for a $25 \mathrm{~nm}(10,10)$-SWNT with the periodic boundary is presented. The energy spectra are computed as,

$$
\begin{aligned}
& E(\omega, k)=\frac{1}{3 n} \sum^{n} \sum_{\alpha}^{3}\left|\frac{1}{N} \int v_{\alpha}(z, t) \exp (i k z-i \omega t) d t d z\right|^{2} \\
& (\alpha=r, \phi, z)
\end{aligned}
$$

where $N$ and $n$ are the number of atoms in the longitudinal (z) direction (the number of unit cells in the nanotube) and number of atoms in a unit cell, respectively. The velocity vector is projected to the local cylindrical coordinates $(r, \phi, z)$ denoted by the subscript $\alpha$ in equation (2). The energy density was firstly computed for each directional component then summed to obtain the overall dispersion relation shown in Fig. 1. Here, $k$-space is normalized by the width of the Brillouin-zone of the SWNT, $\pi / a$, where a is the thickness of a unit cell. In the current case with an armchair SWNT, a unit cell is an armchairshaped monolayer hence $a=\sqrt{3} A_{c-c}$, where $A_{c-c}$ is the interatomic distance. The data are discrete due to the finite length of the nanotube and the broadening of the spectral peaks indicates the phonon scattering. The overall feature of dispersion relations obtained from MD simulations agrees with the reported theoretical models $[1,18]$, especially well with the mechanical model of Mahan and Jeon [18]. 


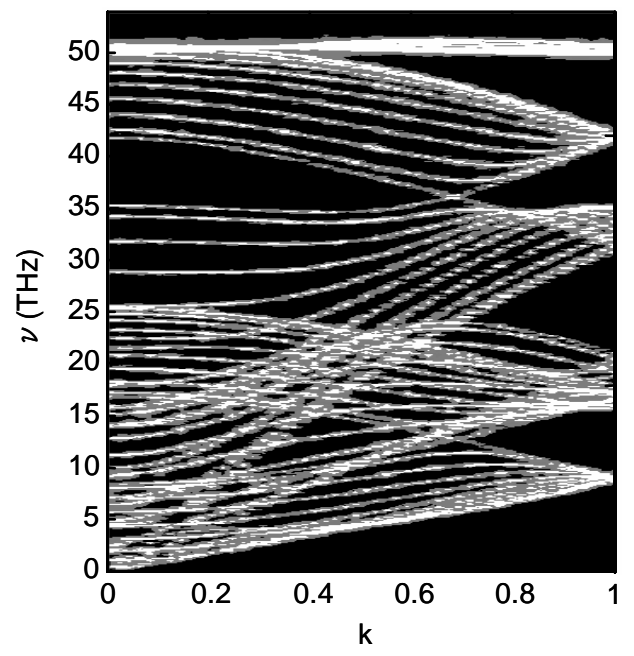

Figure 1. (10, 10)-SWNT PHONON DISPERSION RELATIONS. WAVE NUMBER $k$ IS NORMALIZED WITH THE LENGTH OF BRILLOUIN ZONE.

Note, for SWNTs, as the number of phonon branches is determined by the number of atoms in a unit-cell, even armchair SWNTs whose unit-cell contains fewer atoms than the other structures (chiral and zigzag) with similar diameters, the dispersion relation depicts diverse phonon branches, as seen in Fig. 1 for a $(10,10)-S W N T$. There are optical phonon modes with small circumferential wave number and low frequency that have similar dispersion characteristics and heat capacity to the acoustic ones, especially in the intermediate wavevector $(k)$ regime. Although, acoustic modes are still expected to posses the longest mean free path, the contribution of these optical modes is expected to become important when their mean free paths are comparable to $L$.

\section{RESULTS AND DISCUSSIONS Thermal Conductivity Calculations}

Thermal conductivity $\lambda$ of an SWNT was measured with non-equilibrium MD simulations. After reaching an isothermal state at $300 \mathrm{~K}$ with the auxiliary velocity scaling control, the temperature controlled layers on both ends of the SWNT were activated to apply a temperature difference of $20 \mathrm{~K}$. Eventually the temperature profile converges to a stationary state with quasi-linear gradient. The simulation time ranges within 3-18 ns as the data-convergence time depends on the system size and temperature control methods. By calculating the heat flux along the tube from the energy budgets of the thermostats, $\lambda$ was calculated through the Fourier's law $q=-\lambda d T / d z$. The crosssectional area $A$ of a nanotube was defined using the ring of van der Waals thickness $\pi b d$, where $b=0.34 \mathrm{~nm}$ is van der Waals thickness. The usage of thermal conductivity to express the heat conduction of the current system is arguable due the extensive ballistic heat transport. Furthermore, the definition of the area of an isolated SWNT is rather trivial. Although simply

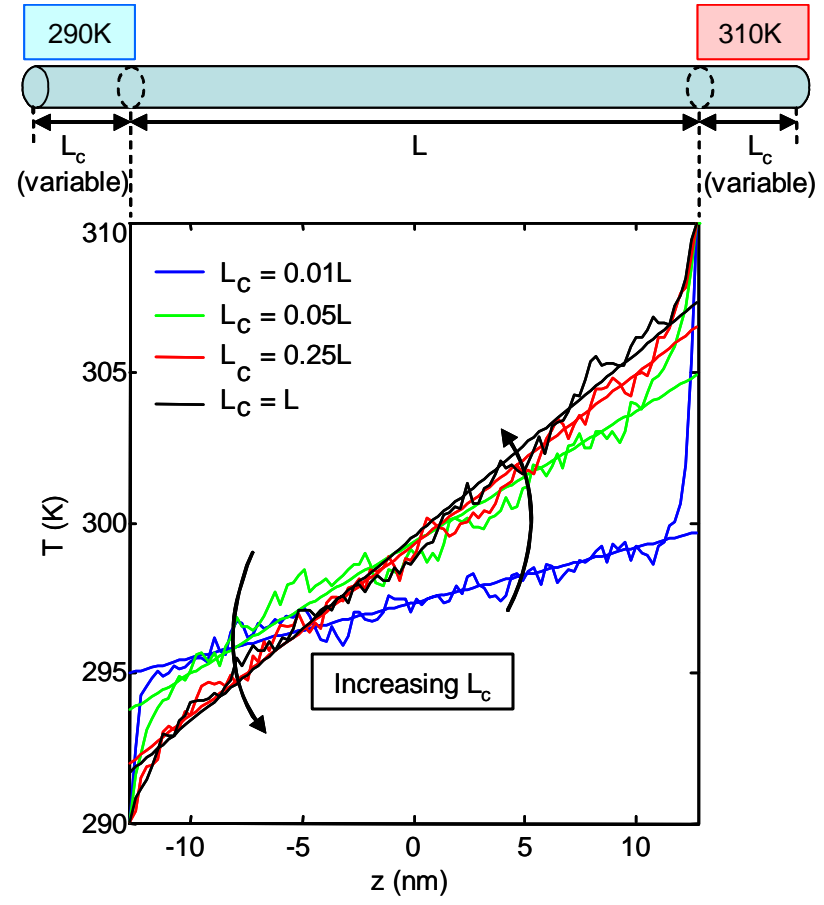

Figure 2. INFLUENCE OF THE LENGTH OF THE LAYERS CONTROLLED BY NOSE-HOOVER THERMOSTAT $\left(L_{C}\right)$ ON THE TEMPERATURE PROFILE $(L=25 \mathrm{~nm})$.

expressing the heat conduction with thermal conductance may be more suitable, here we use thermal conductivity for the sake of comparison with previous studies.

\section{Influence of Thermostats}

On carrying out non-equilibrium MD simulations by applying thermostats to nanotube-ends of an SWNT, the interface between the temperature-controlled part and the rest of the SWNT typically gives rise to a thermal boundary resistance (TBR) $[9,10]$. A TBR appears due to mismatching of lattice-vibrational spectra of the temperature controlled part and the rest of the nanotube. The mismatching causes reflection of phonons and alters scattering dynamics at the interface. Since a TBR is expected to influence the local non-equilibrium phonon distribution and hence alter the thermal conduction, thermostats and their parameters need to be carefully selected to minimize the TBR. In this study, in addition to the phantom technique used in the earlier works [9, 10], we adopt a standard temperature control method using Nose-Hoover (NH) thermostats $[19,20]$.

It is important to state that the TBR effect is not entirely a numerical artifact. In practical use of the lateral heat conduction of SWNTs to promote heat transfer, finite-length SWNTs would be bounded with connections to other materials. In this case, the heat conduction properties would be inevitably altered by TBRs at the connections. Therefore, in fact, it would be more realistic to examine the heat conduction of SWNTs with presence of such interfacial thermal resistance, though 


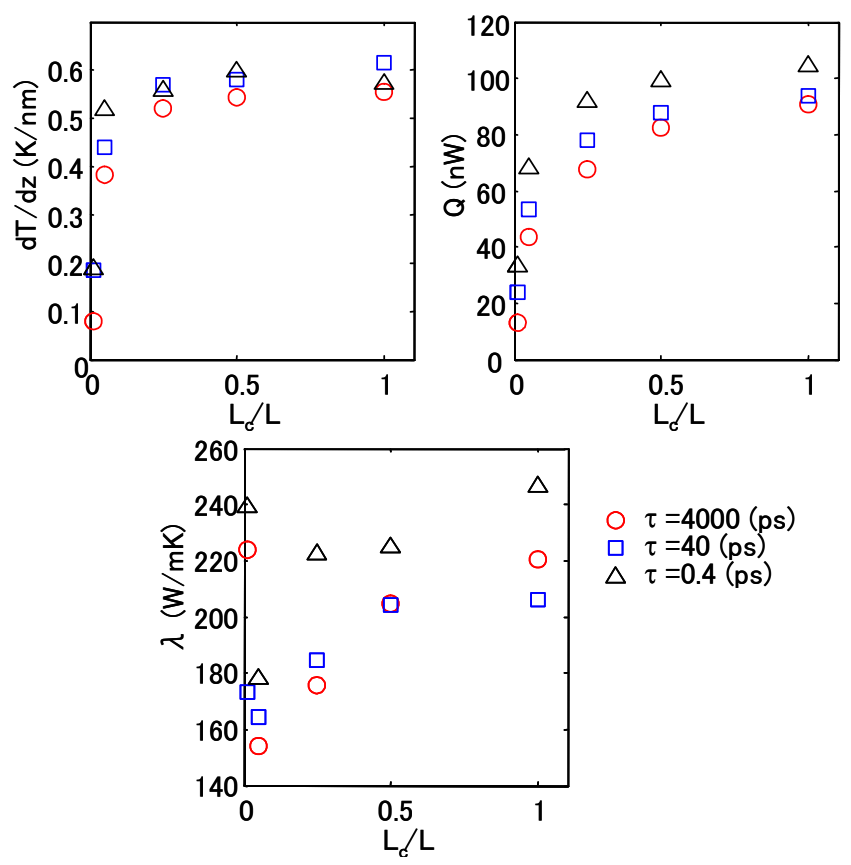

Figure 3. INFLUENCE OF $L_{d} L$ AND RELAXATION TIME OF NOSE-HOOVER THERMOSTAT ON THE AXIAL TEMPERATURE GRADIENT $d T / d z$, HEAT FLUX THROUGH THE SYSTEM $Q$ AND THERMAL CONDUCTIVITY $\lambda$. $L=25 \mathrm{~nm}$.

formulation of a general case would be difficult since such effects would be strongly case-dependent. In the current study, for the sake of comparison with other reported theoretical works and focusing on studying the intrinsic dynamics, we aim to construct an ideal case by minimizing the TBR effect.

Firstly, we carried out thermal conductivity measurements by adopting the phantom technique used in the previous works $[9,10]$. Here, a phantom thermostat consists of a fixed layer and a phantom layer, which are both monolayer unit-cells. Note a unit-cell is an armchair-shaped monolayer in the current case of armchair SWNTs. The phantom layer is placed between the fixed layer (nanotube-end) and the rest of the SWNT and control by the Langevin equation. Debye temperature of diamond was chosen as the damping parameter of the Langevin equation. The formulation aims to damp the phonons traveling into the phantom layer and hence to prevent the phonons from being reflected at the tube ends. Therefore, ideally, a phantom thermostat models isothermal layers with sufficient length.

The simulations using the phantom technique was validated by performing an additional set of simulations adopting a standard application of Nose-Hoover (NH) thermostats. A straightforward application of thermostat without any virtual dynamics makes the method simple and robust, though it is more expensive than the previous method. The thermostats have two tuning parameters; the length of the temperature controlled $L_{c}$ and the relaxation time $\tau$. Fig. 2 shows the temperature profiles obtained by using the $\mathrm{NH}$ thermostat for various values of $L_{c}$, where distinct TBRs can be

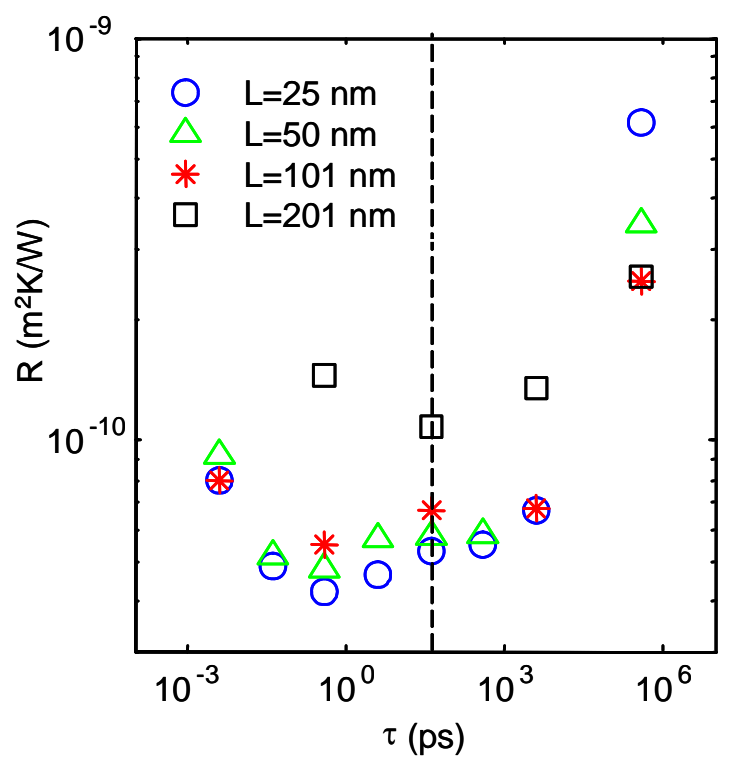

Figure 4. INFLUENCE OF THE RELAXATION TIME OF NOSE-HOOVER THERMOSTAT $\tau$ ON THE SUM OF TBRS ON THE HOT AND COLD SIDES FOR DIFFERENT VALUES OF $L\left(L_{C}=0.5 L\right)$. THE DASHED LINE MARKS $\tau=40 \mathrm{ps.}$

observed as temperature jumps. For instance, in the case of $L_{c}=0.01 \mathrm{~L}$, temperature jumps at the interfaces account for about $50 \%$ of the total temperature difference applied at both tubeends.

In order to minimize the TBRs, parameters $L_{c}$ and $\tau$ were tuned. Influences of $L_{c}$ and $\tau$ on the key thermal properties are described in Fig. 2-4. Elongation of $L_{c}$ permits larger wavelength phonon modes and hence attenuates the discrepancy of phonon spectra between temperature-controlled part and the rest of the nanotube. This can be seen in the $L_{c^{-}}$ dependence of temperature profiles (Fig. 2), where the shorter $L_{c}$ is, the larger TBRs are. More detail views are given in Fig. 3(a-c) which show $L_{c}$-dependences of the temperature gradient, heat flux and thermal conductivity. Both the temperature gradient and heat flux increased with $L_{c}$ and eventually saturated at the upper limit $L_{c} / L \sim 1$, independently of $\tau$. The corresponding trend of $\lambda$ is similar except for the value for $L_{c} / L=0.01$. Therefore, considering the computational cost, we approved $L_{c}=0.5 \mathrm{~L}$ as an optimal value.

As for the relaxation time, longer $\tau$ is expected to give the temperature-controlled layers more time to adjust the spectrum to that of the rest of the SWNT. The variation of TBRs with respect to $\tau$ is shown in Fig. 4 for $L_{c}=0.5 L$. The figure shows the sum of TBRs on the hot and cold sides $R$ for various nanotube lengths, within the parameter bounds ( $40 \mathrm{fs}<\tau<4 \mathrm{~ns}$ ) beyond which the quasi-linear temperature profile is significantly disturbed. Beyond the lower bound, the phonon spectra of temperature-controlled layers and the rest of the SWNT were found to exhibit severe mismatching. On the other hand, beyond the upper bound, the data hardly converged. The figure shows that $R$ takes a minimum value for a critical 


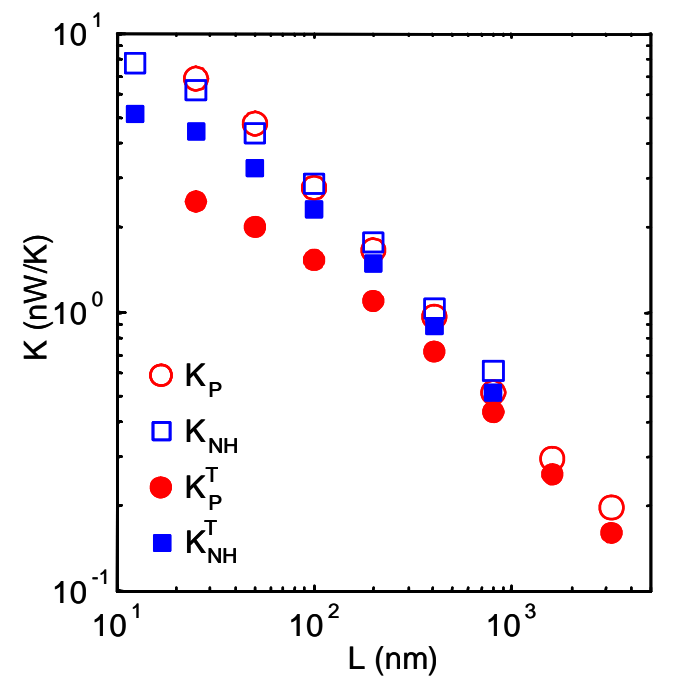

Figure 5. LENGTH DEPENDENCE OF THERMAL CONDUCTANCE $K$ OF $(5,5)$ SWNTS. SUPERSCRIPT T DENOTES THE VALUE FOR THE ENTIRE SYSTEM INCLUDING TBRS. SUBSCRIPTS P AND NH INDICATES THE VALUES COMPUTED USING THE PHANTOM TECHNIQUE AND NOSE-HOOVER THERMOSTATS, RESPECTIVELY.

relaxation time $\tau_{c r}$. On varying $L$ from $25 \mathrm{~nm}$ to $201 \mathrm{~nm}, \tau_{c r}$ exhibits a moderate variation between $400 \mathrm{fs}$ and 40 ps. On considering the general trend where $\tau_{c r}$ increases with $L$, we take $\tau=40 \mathrm{ps}$ as the optimal value. Note that an order difference in $\tau$ may result in approximately $10 \%$ difference in thermal conductivity.

In Fig. 5, the impact of the extent of TBRs on the heat conduction is summarized by plotting the thermal conductance $K=q A / \Delta T$ for $(5,5) \mathrm{SWNTs}$ with lengths. In the figure, subscripts $\mathrm{P}$ and $\mathrm{NH}$ denote the values obtained by phantom techniques and NH thermostats. The open marks are the values calculated by taking the intrinsic temperature drop of SWNTs. The filled marks represent the total thermal conductance calculated based on the total temperature drop $(\Delta T=20 \mathrm{~K})$ including the temperature jumps at the interfaces due to TBRs. Therefore, the differences between the open marks and the corresponding filled marks account for the influence of the TBRs. The magnitude of TBR for the phantom technique is evidently more than that for the case with $\mathrm{NH}$ thermostats. However, difference between the intrinsic thermal conductance $K_{p}$ and $K_{N H}$ is minute. This indicates that the influence of the interfaces on the heat conduction cannot be understood solely by the magnitude of TBRs. The TBR effect decreases with $L$ for both methods since the intrinsic thermal resistance of SWNT increases with $L$. It can be seen that the impact of TBRs on the thermal conductance is minor at the long nanotube limits.

\section{Length Effect on SWNT Thermal Conductivity}

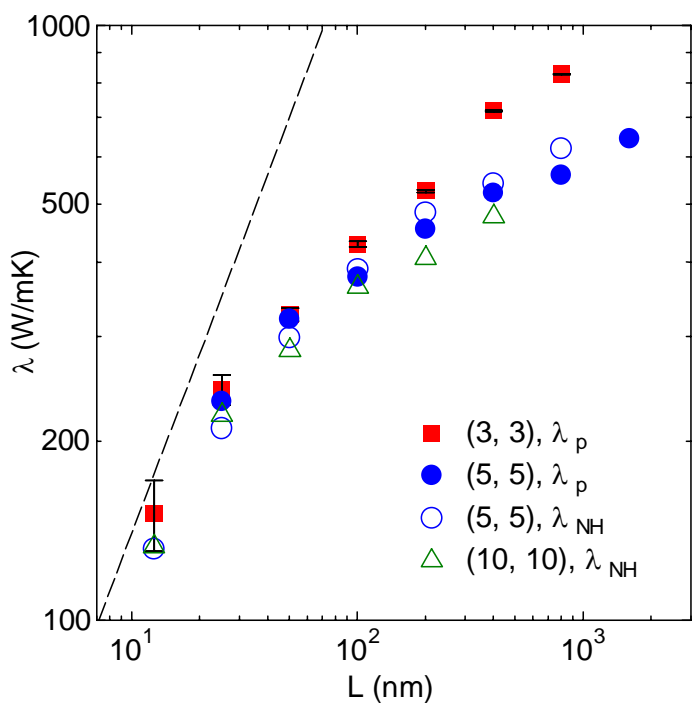

Figure 6. LENGTH AND DIAMETER DEPENDENCES OF SWNT THERMAL CONDUCTIVITY. SUBSCRIPTS P AND NH INDICATE THE VALUES COMPUTED USING THE PHANTOM TECHNIQUE AND NOSE-HOOVER THERMOSTATS, RESPECTIVELY. DASHED LINE IS $\lambda \propto L$ TO INDICATE THE SLOPE IN CASE OF PURE BALLISTIC PHONON TRANSPORT. THE FITTING ERROR BARS ARE DRAWN ONLY FOR THE CASE OF $(3,3)$ SWNTS, WHICH EXHIBITED THE LARGEST ERROR AMONG ALL THE CASES.

In Fig. 6, thermal conductivity of $(5,5)$ SWNTs calculated using phantom technique $\left(\lambda_{P}\right)$ and NH thermostats $\left(\lambda_{N H}\right)$ are marked with circles and asterisks, respectively. Parameters of $\mathrm{NH}$ thermostats are $L_{c}=0.5 \mathrm{~L}$ and $\tau=40 \mathrm{ps}$ as discussed earlier. As mentioned above in terms of thermal conductance, despite the differences in temperature control techniques and intensity of the TBRs, differences between $\lambda_{P}$ and $\lambda_{N H}$ were minor in the examined range of $L$.

Now, let us study the general trend of the length dependence of SWNT thermal conductivity. The overall trend of the slope $(\partial \lambda / \partial L)$ clearly indicates the gradual transition from nearly pure ballistic to diffusive-ballistic phonon transport. When all the phonons experience ballistic phonon transport, $\lambda$ is proportional to $L$ (constant thermal conductance). The asymptotic match of the data to $\lambda \propto L$ (dashed line) suggests nearly pure ballistic phonon transport at the short SWNT limit. Note, on considering the significant phonon population in the a wide range of phonon branches at room temperature, we expect contributions on the heat conduction not only from ballistic transport of acoustic phonon modes but also from that of various optical phonon modes in the small $L$ regime. This is consistent with the results of MD realization of Non-Fourier heat conduction in tens of nanometers long SWNTs, where the ballistic transport of collective optical phonons was observed to play an important role [21]. The gradient $\partial \lambda / \partial L$ gradually decreases as $L$ increases since phonon mean free paths 
gradually become shorter relatively to $L$ i.e. diffusive phonon transport is gradually enhanced with respect to ballistic phonon transport. The positive gradient at the upper bound indicates that the limit of the ballistic phonon transport exceeds a micrometer.

\section{Diameter Effect on SWNT Thermal Conductivity}

Fig. 6 shows diffusive-ballistic thermal conductivity for (3, $3),(5,5)$ and $(10,10)$ SWNTs. For small $L$, where the phonon transport is almost purely ballistic, the thermal conductivity exhibits minor dependence on the diameter. The diameterindependence for small $L$ confirms the above-discussed ballistic phonon transport in this regime. At the ballistic phonon transport limit, where all the populated phonons experience ballistic transport, thermal conductance is proportional to the number of atoms per unit cell, i.e. the diameter if we ignore the variation of the linear phonon transport property (dispersion relations) due to the change in curvature. This means, with the current definition of $A$, that the thermal conductivity is independent of the diameter.

As $L$ increases, the diameter dependence becomes noticeable. As seen in Fig. 6, thermal conductivity profiles of SWNTs with three different chiralities (diameters) deviate beyond $L \sim 100 \mathrm{~nm}$. Here, we assume that the choice of temperature control method has minor influence on the calculated values of thermal conductivity. Current results show that, in the diffusive-ballistic regime, thermal conductivity is larger for SWNTs with smaller diameters, which suggests that the intensity and/or frequency of nonlinear phonon collisions decrease as the diameter decreases i.e. the system becomes closer to one-dimensional structure.

\section{CONCLUSIONS}

Non-equilibrium MD simulations were conducted to investigate the SWNT heat conduction at room temperature in terms of phonon transport. Imposition of a temperature gradient on an SWNT using thermostats typically gives rise to thermal resistances at the boundaries between temperature-controlled layers and the rest of the nanotube, which alter the heat conduction characteristics. By tuning the thermostats to minimize the thermal boundary effect, the length and diameter effects on the thermal conductivity were quantified in a range of $L$ and for three different diameters. The gradual transition from nearly pure ballistic phonon transport to diffusive-ballistic phonon transport was clearly observed. In the small $L$ regime with strong ballistic transport, there is a significant contribution on the heat conduction from a wide range of optical phonons. Corresponding picture of ballistic phonon transport with active role of optical phonons was obtained from the diameter dependence where $\lambda$ is $d$-invariant for small $L$. In the length regime with non-trivial diffusive phonon transport, the diffusion effect due to the phonon collisions is larger for SWNTs with smaller diameter.

\section{ACKNOWLEDGMENTS}

This work is supported in part by the Japan Society for the Promotion of Science for Young Scientists \#1610109 and Grants-in-Aid for Scientific Research \#17656072.

\section{REFERENCES}

1. R. Saito, G. Dresselhaus and M. S. Dresselhaus, Physical Properties of Carbon Nanotubes, Imperial College Press, London (1998).

2. S. Berber, Y-K. Kwon and D. Tomanek, Phys. Rev. Lett. 84, 4613 (2000).

3. C. Yu, L. Shi, Z. Yao, D. Li and A. Majumdar, Nano Lett. 5, 1842 (2006).

4. E. Pop, D. Mann, Q. Wang, K. Goodson and H. Dai 6, 96 (2006).

5. P. Kim, L. Shi, A. Majumdar, and P. L. McEuen, Phys. Rev. Lett. 87, 215502 (2001).

6. M. Fujii, X. Zhang, H. Xie, H. Ago, K. Takahashi, T. Ikuta, H. Abe and T. Shimizu, Phys. Rev. Lett. 95, 065502 (2005).

7. J. Hone, M.C. Llaguno, M. J. Biercuk, A.T. Johnson, B. Batlogg, Z. Benes, J. E. Fischer, Appl. Phys. A 74, 339 (2002).

8. G. Chen, Nanoscale Energy Transport and Conversion, Oxford University Press, New York (2005).

9. S. Maruyama, Physica B, 323, 193 (2002).

10. S. Maruyama, Micro. Therm. Eng., 7, 41 (2003).

11. R. Livi and S. Lepri, Nature, 421, 327 (2003).

12. N. Mingo and D. A. Broido, Nano Lett. 5, 1221 (2005).

13. J. Wang and J-S Wang, Appl. Phys. Lett. 88, 111909 (2006).

14. D. W Brenner, Phys. Rev. B, 42, 9458 (1990).

15. Y. Yamaguchi and S. Maruyama, S., Chem. Phys. Lett., 286, 336 (1998).

16. J. Hone, M. Whitney, C. Piskoti, A. Zettl, Phys. Rev. B. 59, R2514 (1999).

17. T. Yamamoto, S. Watanabe, K. Watanabe, Phys. Rev. Lett. 92, 075502 (2004).

18. G. D. Mahan and G. S. Jeon, Phys. Rev. B, 70, 075405 (2004).

19. S. Nose, J. Chem. Phys., 81 (1), 511 (1984).

20. W. G. Hoover, Phys. Rev. A, 31, 1695 (1985).

21. J. Shiomi and S. Maruyama, Phys. Rev. B 73, 205420 (2006) 